\title{
Depth-controlled laser-induced jet injection for direct three-dimensional liquid delivery
}

\author{
Paul Delrot $^{1}\left[\right.$. Sylvain P. Hauser ${ }^{1}$. Jan Krizek ${ }^{1} \cdot$ Christophe Moser $^{1}$
}

Received: 21 June 2018 / Accepted: 11 August 2018 / Published online: 16 August 2018

(c) The Author(s) 2018

\begin{abstract}
Needle-free jet injection enables the delivery of drugs into skin or soft tissue by puncturing them with a high-velocity liquid jet. However, precise and efficient drug delivery requires generating such liquid jets with both a controlled velocity and a high throughput, which remains challenging with current spring- and gas-actuated jet injectors. Here, we propose a depth-controlled and high-throughput injection method by adapting laser-induced forward transfer (LIFT), a high-resolution two-dimensional printing technique, for direct three-dimensional liquid delivery into soft tissues. The velocity of thin liquid jets is laser actuated from 10 to $85 \mathrm{~m} / \mathrm{s}$ so that doses as small as $10 \mathrm{pL}$, not achievable with other injectors, are injected at a $1 \mathrm{~Hz}$ repetition rate into a $300 \mu \mathrm{m}$ thick soft gelatin substrate with a $25 \mu \mathrm{m}$ depth precision and $12 \mu \mathrm{m}$ lateral resolution. We further investigate the potential of this liquid delivery technique as a direct three-dimensional cell-delivery vehicle and show that depth-controlled particle delivery requires high-delivery efficiency. Our direct three-dimensional liquid delivery system opens up more possibilities for pinpoint drug delivery in soft tissues or tissue-engineered constructs.
\end{abstract}

\section{Introduction}

Needle-free jet injectors generate high-velocity liquid streams, typically ranging from 150 to $300 \mu \mathrm{m}$ in diameter, to puncture soft tissues and deliver drugs for applications as mass immunization or glycemic control [1,2].

Though its small jet diameter potentially makes jet injection less invasive than the $640 \mu \mathrm{m}$ diameter of conventional $23 \mathrm{G}$ hypodermic needles, this needle-free injection method has not gained wide acceptance yet [1,3-5]. This limited acceptance primarily stems from the common pain and bruising induced by uncontrolled injection depth and liquid dispersion into skin or tissue. Indeed, many existing springand gas-actuated jet injectors operate at a single velocity, usually between 100 and $200 \mathrm{~m} / \mathrm{s}$ [2]. This does not allow adapting to the broad variety of mechanical properties of skin and soft tissue in the population, thus occasionally

Electronic supplementary material The online version of this article (https://doi.org/10.1007/s00339-018-2030-6) contains supplementary material, which is available to authorized users.

Paul Delrot

paul.delrot@epfl.ch

1 Laboratory of Applied Photonics Devices, School of Engineering, École Polytechnique Fédérale de Lausanne, 1015 Lausanne, Switzerland resulting in uncontrolled drug delivery into deeper layers and excitation of nerve endings [4, 6-8].

To improve control over the injection depth and delivered dose, several groups have recently developed needle-free jet injectors able to dynamically actuate the jet velocity [4] or jet pressure [7] during the injection. This new generation of jet injectors first produces a brief powerful jet to reach the targeted layer and then a longer jet, below the skin penetration threshold, to deliver a defined dose. These devices are based on a complete redesign of standard jet injectors to operate over a wider range of velocities $(60-160 \mathrm{~m} / \mathrm{s})$ [4] or pressure (5-50 MPa) [7], which was respectively achieved with a piezo- and Lorentz-force actuator.

Another approach to reduce injection-associated pain consists in producing thinner jets $(50-100 \mu \mathrm{m})$ that penetrate in shallower skin's layers $(100-200 \mu \mathrm{m})[9,10]$, which is sufficiently deep to cross the greatest barrier to drug transport but shallow enough to avoid the most innervated layers [11]. Such microjet injectors were designed to run in a repetitive $[9,10]$ way through piezo-actuation, and studies demonstrated that this approach allows volumes with a dose precision in the nanoliter range $(2-30 \mathrm{~nL})$ to be delivered. Doses could further be injected locally at a frequency larger than $1 \mathrm{~Hz}$ within an area $>100 \mu \mathrm{m}$ in diameter, which we define as the lateral resolution of injection. 
Improving the lateral resolution of injection would open up possibilities to study the local effect of drug or growth factor delivery, for instance into tissue-engineered constructs for drug screening applications or bio-engineering [12]. Recently, a $30 \mu \mathrm{m}$ lateral resolution was obtained for the injection of nanoliter doses into a soft tissue model [13]. This was achieved using a laser-actuated needle-free microjet injector, in which a laser-induced shockwave propels a liquid through a nozzle. However, as this precise laseractuated microjetting method is nozzle-based, it requires a reloading step between each injection, which limits its throughput or demands specific engineering efforts for designing an automatic reloading feature [7].

Laser-induced forward transfer (LIFT) could potentially overcome this limitation while improving the achievable lateral resolution of injection and dose precision. In standard LIFT, a thin layer $(10-100 \mu \mathrm{m})$ of the liquid to deliver is coated onto a transparent donor slide. Upon absorption of a nanosecond laser pulse by an absorptive layer of the donor slide, a shockwave is generated, which produces a liquid microjet towards a receiver slide [14-17]. Thus, LIFT can directly deliver biologically relevant liquids over the two dimensions of the donor slide without any reloading steps. In addition, LIFT enables liquid microjets to be generated over a wide range of velocity by varying the incident laser fluence on the donor slide [18, 19].

In this paper, we propose to further improve the lateral resolution, dose precision and throughput of current needle-free microjet injectors by adapting LIFT for direct three-dimensional liquid delivery. With this aim in mind, we exploit the velocity range of LIFT microjets to achieve depth-controlled injection of $10 \mathrm{pL}$ doses within a $300 \mu \mathrm{m}$ thick soft gelatin tissue model. The injections are performed over a 2.8 by $1.5 \mathrm{~mm}$ area of the LIFT donor slide, thus demonstrating direct three-dimensional liquid delivery. We investigate the dependence of the injection depth with respect to the jet velocity, as well as the delivery efficiency of our new delivery method. We further study the potential of our method as a direct three-dimensional cell-delivery vehicle.

\section{Materials and methods}

\subsection{LIFT for direct three-dimensional liquid delivery}

Direct three-dimensional liquid delivery is achieved by adapting a two-dimensional LIFT system developed by Fraunhofer-ILT (LIFTSYS). The solid receiver slide typically used in standard LIFT systems is replaced by a 300 $\mu \mathrm{m}$ thick soft gelatin substrate so that microjets are directly injected from the donor slide into this soft tissue model (see Fig. 1a). The donor slide and the top surface of the gelatin substrate are placed $1400 \mu \mathrm{m}$ apart. Both the donor slide and receiver substrate are carried in the LIFTSYS machine by two-axis stages for two-dimensional lateral motion and patterning.

The donor slide (see top of Fig. 1a) consists of a standard silica microscope slide onto which a $60 \mathrm{~nm}$ light-absorptive titanium layer is sputtered (Alliance Concept, DP650). A $30 \mu \mathrm{m}$ thick layer of liquid ink is then coated with a doctor blade (Elcometer, model 3580) onto this titanium layer. The donor slide is then placed in the LIFTSYS machine where $5 \mathrm{~ns}$ UV laser pulses are absorbed by the titanium layer, thereby vaporizing part of the titanium layer and of the ink. The expansion of the resulting high pressure vapor bubble pushes the ink forward, which in turn produces a liquid microjet of ink [20,21].

The liquid ink is made of $1 \%(\mathrm{w} / \mathrm{v})$ sodium alginate (Sigma-Aldrich) into a 1:3 (v/v) mixture of glycerin (> $99.5 \%$, Roth) and deionized water (density $1.1 \mathrm{~g} \mathrm{~mL}^{-1}$ ). To image the depth and morphology of injection, $0.2 \mu \mathrm{m}$ fluorescent beads (Thermo Fischer, FluoSpheres ${ }^{\circledR}$ F8811) are added to the ink with a concentration of $5 \times 10^{9}$ beads/ $\mathrm{mL}$. For the experiments investigating the potential of our technique as a direct three-dimensional cell-delivery vehicle, cell-like fluorescent beads $\left(\varnothing 10 \mu \mathrm{m}\right.$, density: $1.05 \mathrm{~g} \mathrm{~mL}^{-1}$, Polysciences, Fluoresbrite ${ }^{\circledR}$ YG) were also added to the ink with a concentration of $1 \times 10^{7}$ beads $/ \mathrm{mL}$, similar to cell concentrations used in previous studies on cell delivery using LIFT [16, 22].

Previous studies on LIFT systems used either solid receiver substrates when patterning non-sensitive material [21], or receiver slides coated with a thin layer of damping material such as Matrigel or gelatin to increase cell viability for bioprinting applications [23-25]. Here, to investigate direct three-dimensional liquid delivery, the receiver substrate consists of a $300 \mu \mathrm{m}$ thick soft gelatin substrate. Gelatin, as an inert and biocompatible material [26], has been used as a soft tissue model [3, 13, 27] or as a scaffold material for soft tissue-engineering applications [28-30]. Interestingly, gelatin exhibits Bingham plastics rheological properties. As shown in the rheological analysis of Fig. 1b, above a threshold shear stress, the yield stress, gelatin flows as a liquid whereas it behaves as a solid below this yield stress [31]. In terms of jet dynamics, this means that above a threshold jet velocity, gelatin will be punctured and the jet will flow into it. Once the drag force has sufficiently slowed down the jet, gelatin will act as a solid support material for the injected dose.

To perform confocal imaging of the injections, we used coverslip-mounted plastic dishes (MatTek, model P35G-1.514-C) as gelatin containers (receiver substrates). A solution of $2 \%(\mathrm{w} / \mathrm{v})$ gelatin (Sigma-Aldrich, $300 \mathrm{~g}$ bloom force) and $0.5 \%(\mathrm{w} / \mathrm{v}) \mathrm{CaCl}_{2}$ (Sigma-Aldrich) in deionized water was prepared at $37{ }^{\circ} \mathrm{C}$ and mixed with $0.2 \mu \mathrm{m}$ fluorescent beads 


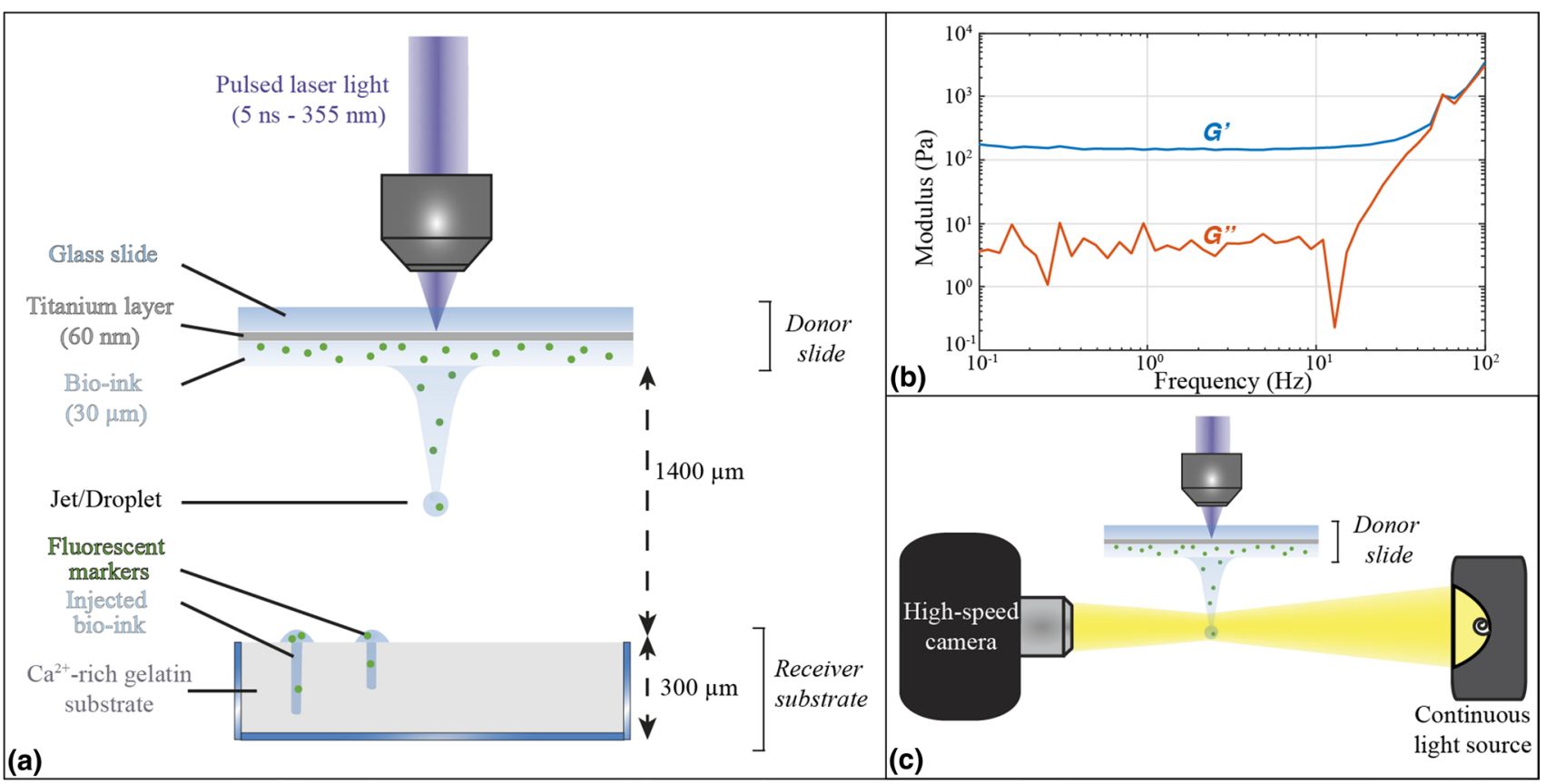

Fig. 1 a Experimental setup for three-dimensional liquid delivery by laser-induced forward transfer. Liquid bio-ink jets are generated from the donor slide at different velocities depending on the laser fluence. As their impact velocity increases, these liquid jets penetrate deeper into the gelatin receiver substrate. b Rheological analysis of elas- tic $\left(\mathrm{G}^{\prime}\right)$ and viscous $\left(\mathrm{G}^{\prime \prime}\right)$ modulus for the gelatin receiver substrate showing Bingham plastic behavior. c Experimental setup for timeresolved imaging of the laser-induced jets of bio-inks. Jets are illuminated by a strong continuous white light source and imaged using a high-speed camera mounted with a microscope objective
(Thermo Fischer, FluoSpheres® F8810) at a concentration of $5 \times 10^{9}$ beads $/ \mathrm{mL}$. Labeling the gelatin substrate (density $1.3 \mathrm{~g} \mathrm{~mL}^{-1}$ ) with fluorescent markers orthogonal to that of the injected liquid allowed us to determine precisely the top surface of the gelatin volume and the injection depth. The liquid mixture was then poured in the plasma-cleaned dish to form a $300 \mu \mathrm{m}$ thick volume with a flat top surface. The receiver substrates were stored at $5{ }^{\circ} \mathrm{C}$ at least for $2 \mathrm{~h}$, until experiments took place. The experiments were run at $22^{\circ} \mathrm{C}$ and lasted 4 min thus not degrading the gelatin's rheological properties, after which the receiver substrates were stored back at $5{ }^{\circ} \mathrm{C}$ until being imaged. As mentioned above, the injections were imaged with a confocal fluorescent microscope under a protected atmosphere $\left(22^{\circ} \mathrm{C},>85 \%\right.$ humidity) to prevent drying of the samples. Finally, the image tiles were reconstructed using an open-source plugin [32].

\subsection{Time-resolved imaging of jet velocity}

The jet dynamics were imaged using a custom-made highbrightness continuous light source combined with a highspeed camera (Vision Research, Phantom Miro M310) mounted with a microscope objective. The LIFTSYS machine allowed us to image the jetting velocity near the donor slide. However, the small space available within the machine did not allow us imaging the impact of the jets onto the soft gelatin substrates (see Fig. 1c).

\section{Results and discussion}

\subsection{Controlling the jetting velocity with the laser fluence}

Precise and efficient needle-free drug delivery requires controlling the injected dose, its dispersion, which we define as the lateral resolution, as well as its injection depth. More specifically, depth-controlled needle-free injections demand the production of liquid microjets over a wide range of velocity, which was previously achieved by dynamic piezo[4] or Lorentz-force actuation [7].

Here, by varying the incident laser fluence on the LIFT donor slide (see Fig. 1a), we demonstrate microjet generation with estimated velocities at impact ranging from $10 \mathrm{~m} / \mathrm{s}$ to more than $80 \mathrm{~m} / \mathrm{s}$ (see Fig. 2a). The estimated velocity at impact on the substrate is measured $1400 \mu \mathrm{m}$ away from the donor slide, where the top surface of the soft gelatin substrate stands during injection experiments (see the dashed lines in Fig. 2b-d).

Interestingly, our device allows generating stable jets, with a high directionality over this wide range of velocities 


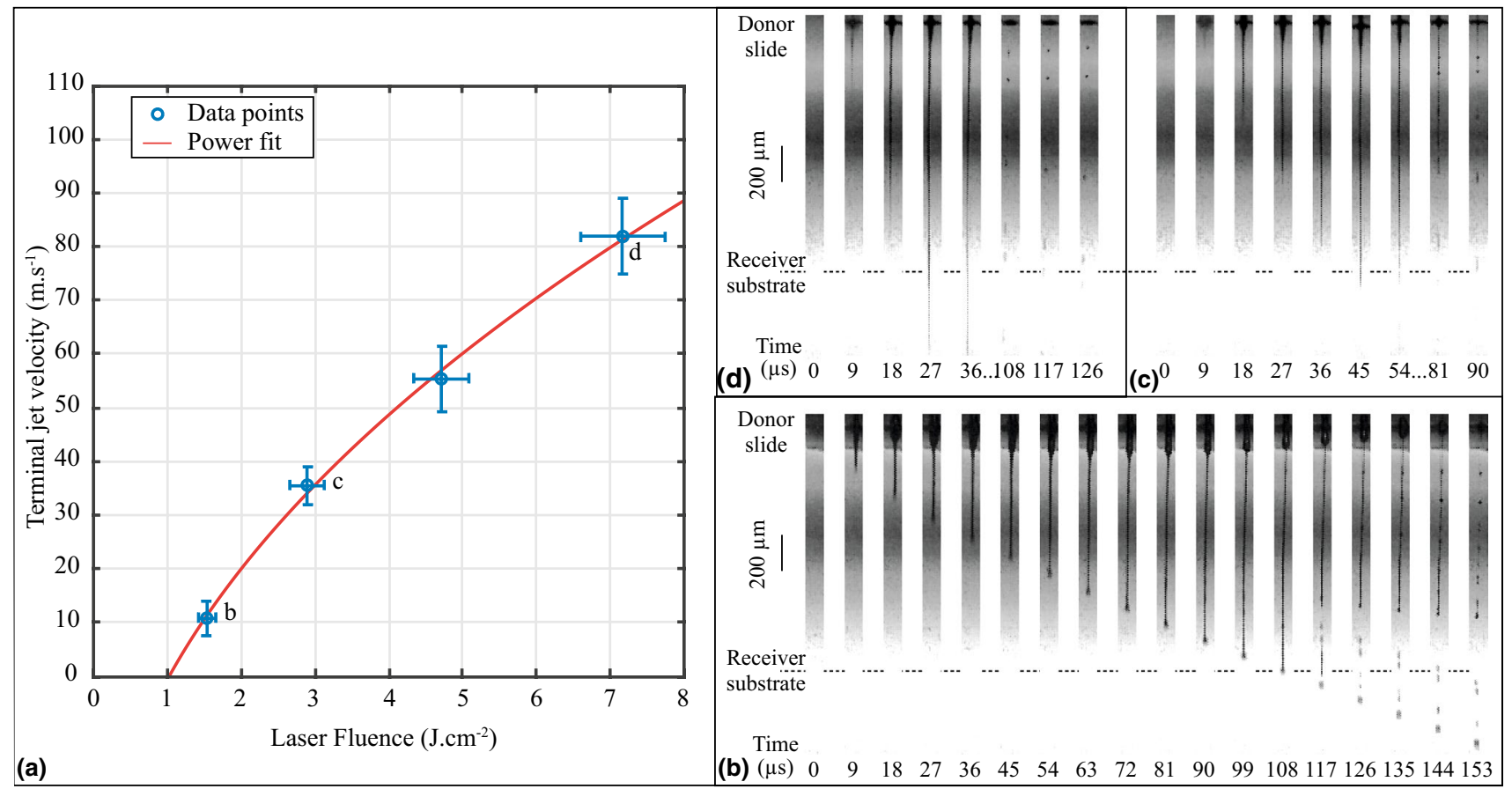

Fig. 2 a Jet velocity at $1.4 \mathrm{~mm}$ from the donor slide as a function of the incident laser fluence on the donor slide. $\mathbf{b}$ Time-resolved imaging of the laser-induced jet for a $1.53 \pm 0.12 \mathrm{~J} \mathrm{~cm}^{-2}$ laser fluence. c $2.89 \pm 0.23 \mathrm{~J} \mathrm{~cm}^{-2}$ laser fluence. d $7.17 \pm 0.57 \mathrm{~J} \mathrm{~cm}^{-2}$ laser fluence. Low-velocity liquid droplets or trailing filaments can be observed for

(see Fig. 2b-d and supplemental material for movies V1, $\mathrm{V} 2$ and V3), whereas standard jet injectors typically create turbulent jets [1] which can degrade the lateral resolution of injection. In the same way, as shown in Figs. 2b-d, our LIFT system enables the production of these high-speed jets with a width as small as $\sim 15 \mu \mathrm{m}$, which also contributes to a finer lateral resolution of injection (see Sect. 3.2) than existing needle-free microjet injectors $[10,13]$.

LIFT jets could potentially be produced over an even larger velocity range by increasing the ink's viscosity, as viscosity tends to stabilize laser-induced jet and droplet generation [33, 34]. However, for this study the LIFTSYS's laser experimentally limited us to an upper fluence limit of $7.17 \pm$ $0.57 \mathrm{~J} \mathrm{~cm}^{-2}$, therefore the stability of higher velocity jetting could not be investigated.

\subsection{Depth-controlled injection for direct three-dimensional liquid delivery}

To assess the ability of our device to achieve needle-free depth-controlled injections we use the setup of Fig. 1a with the unseeded ink described in Sect. 2.1, and generate grids of 11 by 20 jets, each row of jets being injected with an increasing laser fluence and therefore with an increasing jet velocity (see Fig. 2a). times $>99 \mu \mathrm{s}, 81 \mu \mathrm{s}$ and $>108 \mu \mathrm{s}$ in $\mathbf{b}-\mathbf{d}$, respectively. These low velocity volumes are responsible for the liquid deposited on top of the injection sites (see Fig. 3a). The sequences of $\mathbf{b}-\mathbf{d}$ were acquired at $\sim$ $100,000 \mathrm{fps}$ and with a $6 \mu$ s exposure

Above a threshold velocity, the gelatin substrate is punctured and the injection depth is reproducibly controlled from 0 to $\sim 230 \mu \mathrm{m}$, linearly scaling with the jet velocity (see red data points and dashed fit in Fig. 3a). This linear trend is consistent with the viscous stress model developed by Tagawa et al. [13] in which a viscous drag force, proportional to the jet velocity $v$, is applied to the liquid as it penetrates into the material. The injection depth $D_{\mathrm{p}}$ then linearly scales with the jet velocity $v$ as:

$D_{\mathrm{p}}=\frac{1}{c_{\mathrm{v}}}\left(v-v_{\mathrm{c}}\right)$

where $c_{\mathrm{v}}$ is a fitting parameter in $\mathrm{s}^{-1}$ and $v_{c}$ is the threshold velocity for puncturing the material [13], which we find to be $v_{c}=7.5 \mathrm{~m} / \mathrm{s}$ by fitting our data with this viscous stress model. Such a threshold model is also consistent with the Bingham plastic behavior measured for the gelatin substrate (see Fig. 1b) since below a yield velocity, the material remains solid to the impact of the jet whereas above this yield velocity, it flows under impact.

The depth precision of the injection is $\pm 25 \mu \mathrm{m}$ (see red error bars in Fig. 3a) while the lateral precision of delivery is $\pm 4 \mu \mathrm{m}$, which we derive from the deviation of the experimental injection positions from the targeted injection positions over 200 jet injections (not depicted here). In addition, 
(a)

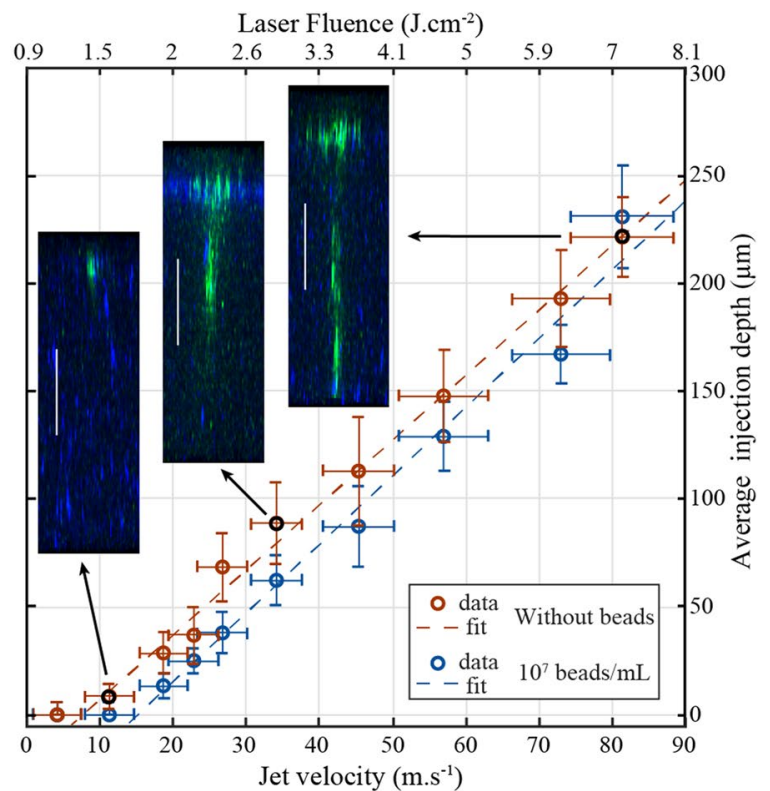

(b)

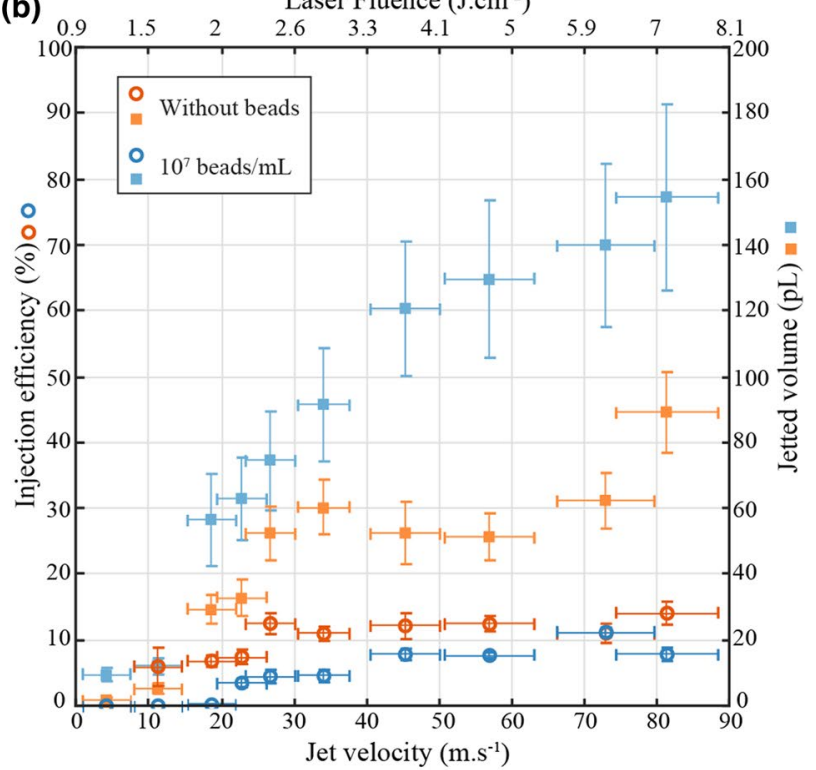

Fig. 3 a Average injection depth as a function of the jet velocity (bottom $x$-axis) and the incident laser fluence on the donor slide (top $x$-axis). The data points of both the plain ink and the particle-seeded ink are linearly fitted to show consistency with a viscous stress model [13]. Cross-sections of the injected ink are depicted for various data points of the plain ink, scale bar $100 \mu \mathrm{m}$, green: injected liquid, blue: soft gelatin substrate (each data point is the average of 20 experiments). b Average injection efficiency (blue and red circles) and jetted volume (blue and red squares) as a function of the jet velocity and laser fluence (each data point is the average of 20 experiments)

the lateral resolution of injection of our device, which we define as the lateral width over which the delivered liquid is dispersed, is $12 \pm 4 \mu \mathrm{m}$ (see cross-sections of injection sites in Fig. 3a). This low dispersion of the delivered liquid is likely due to the ultra-thin microjets generated by our LIFT system (see Figs. 2b-d) rather than the gelation of the alginate-based ink in the $\mathrm{Ca}^{2+}$-rich gelatin substrate. Analyzing the respective penetration time of the jet and gelation time of the alginate-based ink reveals indeed that the injection occurs over a time shorter by several orders of magnitude, $\sim 3 \mu$ s, than the diffusion [35] of calcium ions over $6 \mu \mathrm{m}$, $\sim 30 \mathrm{~ms}$.

Similarly, the geometry of micro-injections shown in the imaging cross-sections of Fig. 3a, with a thin injected column of ink capped with a larger volume on the gelatin surface, differs from the usual puncture and dispersion profile of existing needle-free microjet injectors $[9,10,13]$. This difference might result from the stiffer injected materials used in these previous studies, whose stiffness tend to disperse sideways the injections.

A possible backflow of the injected liquid towards the substrate surface could also account for both the low dispersion of injection and the presence of the larger volume of liquid on the substrate surface, respectively, because backflow could reduce the ability of the microjet to induce further cracks in the gelatin and because liquid could flow back towards the gelatin's surface [36]. However, such a backflow model would experimentally be evidenced by an asymptotic depth of injection resulting from an insufficient kinetic energy of the jet to induce further material failure [36]. As we do not observe this asymptotic depth of injection in Fig. 3a, we rather speculate that the volume of liquid appearing on the top surface of injection (see imaging cross-sections of Fig. 3a) is due to the low-velocity trailing filament and satellite droplets produced at the end of the LIFT jetting process (see images for times $>99 \mu \mathrm{s}, 81 \mu \mathrm{s}$ and $>108 \mu$ s in Fig. $2 b-d$ respectively).

These low-velocity components of the laser-induced jet also highly degrade the delivery efficiency of our system, that is to say the ratio of injected volume over the total jetted volume. Here, as the jetted volume was measured by fluorescent imaging of the injection sites after each experiment (see Sect. 2.1), the volume of ink that goes back towards the donor slide (see Fig. 2c) could not be measured and its contribution to the total jetted volume was neglected.

The delivery efficiency quickly reaches an asymptotic value of $\sim 12 \%$ once the jet overcomes the threshold velocity for puncture, as respectively shown with red circles in Fig. 3a, b. This delivery efficiency is relatively low compared to recent works on needle-free microjet injections in soft samples, where the authors typically demonstrated $90 \%$ efficiency $[10,13]$. Improving the delivery efficiency of our device would require to either impart the same velocity to the whole body of jetted liquid or to suppress its low velocity components, namely the trailing filament and satellite droplets (see Fig. 2b-d). These two conditions are equivalent to jetting a single high-velocity droplet. Recent studies $[15,37]$ showed that single-droplet production can be 
achieved through LIFT by tuning the ink's viscoelastic properties. A dimensionless parametric study suggests that our ink is too elastic for single-droplet jetting (see supplemental material for further details on the dimensionless parametric study), which we will study in future work.

Though our LIFT microjet injector currently has a relatively low delivery efficiency, it allows injecting picoliter doses (see red squares in Fig. 3b) over a two-dimensional area with $\mathrm{a} \pm 25 \mu \mathrm{m}$ depth control as well as a $\pm 4 \mu \mathrm{m}$ lateral precision and a $12 \pm 4 \mu \mathrm{m}$ lateral dispersion. This micrometric lateral and axial control over the liquid delivery is comparable to the size of a cell and therefore falls within the range of the optimal printing precision for direct three-dimensional delivery of cells or particles [12]. We thus investigate in the next section the ability of our LIFT system to deliver particles at a pinpoint location in a volume.

\subsection{Application to particle delivery}

LIFT, as a precise biocompatible printing system, has been extensively used in the past years for two-dimensional cell patterning or to build layer-by-layer three-dimensional tissue engineered constructs $[17,38,39]$. Needle-free depthcontrolled delivery of cells or particles within soft tissues or support material would, for instance, enable studying cell responses to specific micro-architectures in a minimally invasive way. To study the potential of our direct threedimensional LIFT injector to carry a cell-like payload, we seed the liquid ink of the donor slide with $\varnothing 10 \mu \mathrm{m}$ fluorescent beads (see Sect. 2.1 for further details).

The particle-seeded liquid is injected less deep into the soft gelatin substrate than the plain liquid (see blue and red datapoints respectively in Fig. 3a), a phenomenon already observed in previous work for larger doses [5]. Here, as evidenced by the poorer fit to the viscous stress model (see blue dashed line in Fig. 3a) compared to the plain liquid, we hypothesize that the seeded particles influence the puncture ability of the jet. The particles are indeed approximately the same size, $\varnothing 10 \mu \mathrm{m}$, as the jet thickness, $\sim 12 \mu \mathrm{m}$, which could affect the jet cross-sectional drag area, thus reducing its potential for puncture and penetration.

The delivery efficiency of the particle-seeded liquid is almost halved compared to the plain liquid, reaching an asymptotic value of $\sim 7 \%$ (see blue circles in Fig. 3b), which is caused by the larger amount of liquid jetted with the particle-seeded liquid than with the plain liquid (see blue and orange squares in Fig. $3 \mathrm{~b}$ ). We speculate that a larger volume of low-velocity liquid is pulled towards the substrate by bead aggregates, possibly induced by capillary forces between beads. This hypothesis is supported by the higher average number of beads deposited per dose than what would be expected from the initial bead concentration in the liquid. As shown in Fig. 4, fitting the experimental

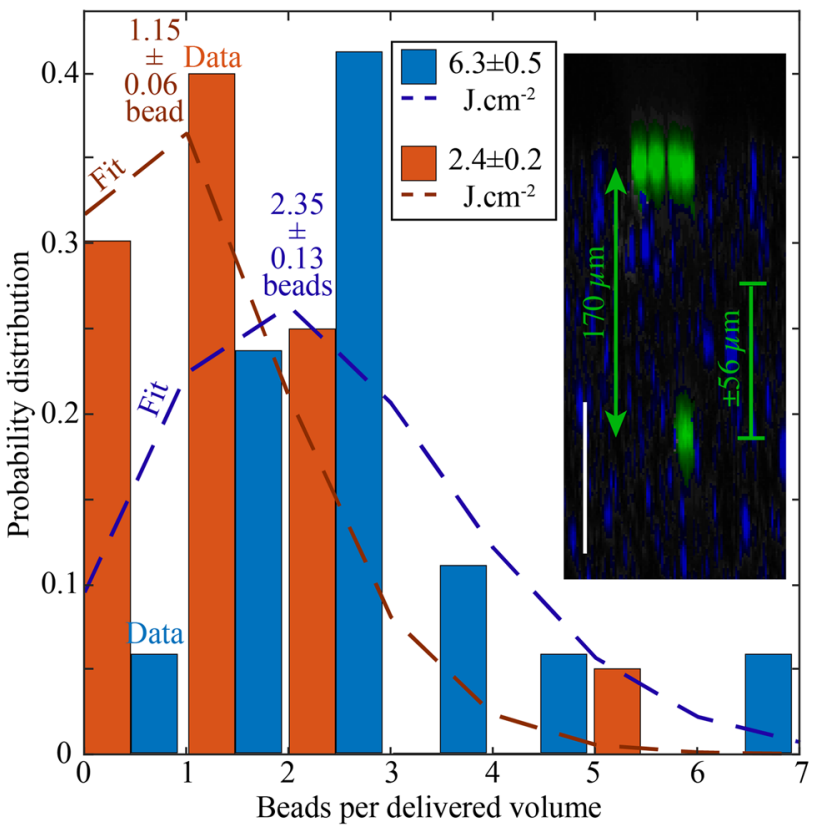

Fig. 4 Probability distribution of the amount of beads per delivered dose for two laser fluences. The orange and blue bar plots, respectively, represent the measured distribution of delivered beads for doses jetted at $2.4 \pm 0.2 \mathrm{~J} \mathrm{~cm}^{-2}$ and $6.3 \pm 0.5 \mathrm{~J} \mathrm{~cm}^{-2}, \mathrm{~N}=20$. The dashed orange and blue lines are Poisson distribution fits to the experimental bead distribution. These fits show that an average of $1.15 \pm 0.06$ bead and $2.35 \pm 0.13$ beads are delivered at $2.4 \pm 0.2 \mathrm{~J} \mathrm{~cm}^{-2}$ and $6.3 \pm 0.5 \mathrm{~J} \mathrm{~cm}^{-2}$, respectively, which is compared to the $0.75 \pm 0.15$ bead and $1.4 \pm 0.22$ bead derived from the measured jetted volume (see Fig. $3 \mathrm{~b}$ ) and the bead concentration in solution. A cross-section of injection with the seeded ink is shown on the right, several beads (in bright green) are deposited on the top gelatin surface (in blue) while one bead is injected $\sim 170 \mu \mathrm{m}$ into the substrate. The green deviation bar represents the depth uncertainty on the deposition of beads for a laser fluence of $7.2 \pm 0.6 \mathrm{~J} \mathrm{~cm}^{-2}$. The image is processed to better distinguish the deposited beads. White scale bar $100 \mu \mathrm{m}$

bead distributions by Poisson distributions [23] reveals that an average $1.15 \pm 0.06$ and $2.35 \pm 0.14$ beads per dose are, respectively, delivered for $2.4 \pm 0.2$ and $6.3 \pm 0.5 \mathrm{~J} \mathrm{~cm}^{-2}$ laser fluences, whereas combining the measured dose volume (see blue squares in Fig. 3b) with the $10^{7}$ beads $/ \mathrm{mL}$ concentration indicates that $0.75 \pm 0.15$ and $1.4 \pm 0.22$ bead per dose would, respectively, be expected. This inconsistency suggests that beads are forming aggregates prior or during jetting, and are likely to increase the amount of lowvelocity liquid transferred towards the gelatin substrate.

However, as shown in the cross-section of injection of Fig. 4, we demonstrate that cell-like beads can be injected into a soft gelatin substrate using our LIFT microjet device. The injected beads lie in the $12 \mu \mathrm{m}$ diameter thin column of liquid depicted in Fig. 3a, thus precise lateral delivery of the cell-like payload can be achieved. Since more volume of liquid is delivered on the substrate 
surface than injected into the substrate, the likelihood of bead injection into the substrate proportionally decreased (see Fig. 4). Similarly, this degrades the control over the delivery depth of the particles, as indicated by the green error bar in Fig. 4 that shows that for a laser fluence of $7.2 \pm 0.6 \mathrm{~J} \mathrm{~cm}^{-2}, \mathrm{a} \pm 57 \mu \mathrm{m}$ deviation on the injection depth of beads is measured over 11 experiments, which corresponds to a 33\% relative depth uncertainty. Embedding particles in a single high-velocity droplet would allow overcoming this limitation, which will be studied in future work through modification of the ink rheology as described in Sect. 3.2 and in the supplemental material.

Moreover, in the case of cell-seeded liquids, cell viability should be considered because of the high velocity at impact achieved in our study $\left(10-80 \mathrm{~ms}^{-1}\right)$. LIFT is an established technique for cell deposition, with postprinting cell viability reaching $>95 \%[40,41]$. Furthermore, previous studies showed that cell suspensions could withstand laser-induced acceleration and deceleration of the order of $10^{6}-10^{7} \mathrm{~g}$ and authors speculated that only the cells at the front of the jet were damaged [42, 43]. By analogy, our laser-assisted system could potentially be viable for sensitive cell-seeded liquids as we estimate from time-resolved imaging that the maximum deceleration at impact is of the order of $10^{6} \mathrm{~g}$.

\section{Conclusion}

We demonstrate depth-controlled liquid injection into a 300 $\mu \mathrm{m}$ soft gelatin substrate over two dimensions, thus achieving direct three-dimensional liquid delivery. The injection depth is controlled by producing $15 \mu \mathrm{m}$ stable liquid streams over a wide range of velocities with a LIFT system. This laser-assisted device operates over two dimensions without any reloading steps and enabled us delivering picoliter doses up to depth of $\sim 230 \mu \mathrm{m}$ with a $25-\mu \mathrm{m}$ depth repeatability and a $12 \mu \mathrm{m}$ lateral resolution. Using this system, we investigate needle-free particle injection and show that the current delivery efficiency and the probability distribution followed by the particles limits the depth control to $\pm 57 \mu \mathrm{m}$. The optimization of the liquid rheology to generate high-velocity single-droplet could potentially improve the control over the deposition depth of particles.

The direct depth-controlled delivery of picoliter doses over two dimensions opens up new possibilities to study local effects of drug or growth factor delivery, for instance in tissue-engineering applications [12]. Furthermore, combining more powerful lasers with viscous inks [33] could potentially enable our device to generate faster microjets for needle-free injection into stiffer tissues or materials than the soft gelatin susbtrate used in this paper.
Acknowledgements We thank Prof. J. Brugger (EPFL-LMIS1) for lending us the LIFTSYS machine, as well as J. Pernollet (EPFL-CMi) and Dr. M. Wehner (Fraunhoher-ILT) for technical support on the LIFTSYS machine, and Prof. F. Gallaire (EPFL-LFMI) for lending us the high-speed camera. PD acknowledges Dr. D. Loterie (EPFL-LAPD) for manuscript revision. PD benefited from a grant from EPFL-STI for Advanced Additive Manufacturing research.

Open Access This article is distributed under the terms of the Creative Commons Attribution 4.0 International License (http://creativeco mmons.org/licenses/by/4.0/), which permits unrestricted use, distribution, and reproduction in any medium, provided you give appropriate credit to the original author(s) and the source, provide a link to the Creative Commons license, and indicate if changes were made.

\section{References}

1. S. Mitragotri, Innovation - current status and future prospects of needle-free liquid jet injectors. Nat. Rev. Drug Discov. 5, 543 (2006)

2. A. Arora, M.R. Prausnitz, S. Mitragotri, Micro-scale devices for transdermal drug delivery. Int. J. Pharm. 364, 227 (2008)

3. T. Kato, T. Arafune, T. Washio, A. Nakagawa, Y. Ogawa, T. Tominaga, I. Sakuma, E. Kobayashi, Mechanics of the injected pulsejet into gelatin gel and evaluation of the effect by puncture and crack generation and growth. J. Appl. Phys. 116, 074901 (2014)

4. J.C. Stachowiak, T.H. Li, A. Arora, S. Mitragotri, D.A. Fletcher, Dynamic control of needle-free jet injection. J. Control Release 135, 104 (2009)

5. Y. Michinaka, S. Mitragotri, Delivery of polymeric particles into skin using needle-free liquid jet injectors. J. Control Release 153, 249 (2011)

6. J. Schramm, S. Mitragotri, Transdermal drug delivery by jet injectors: energetics of jet formation and penetration. Pharm. Res. 19, $1673(2002)$

7. B.D. Hemond, A. Taberner, C. Hogan, B. Crane, I.W. Hunter, Development and performance of a controllable autoloading needle-free jet injector. J. Med. Dev. Trans. ASME 5, 015001 (2011)

8. E.E. Kis, G. Winter, J. Myschik, Devices for intradermal vaccination. Vaccine 30, 523 (2012)

9. A. Arora, I. Hakim, J. Baxter, R. Rathnasingham, R. Srinivasan, D.A. Fletcher, S. Mitragotri, Needle-free delivery of macromolecules across the skin by nanoliter-volume pulsed microjets. Proc. Natl. Acad. Sci. USA 104, 4255 (2007)

10. A.M. Römgens, D. Rem-Bronneberg, R. Kassies, M. Hijlkema, D.L. Bader, C.W.J. Oomens, M.P.B. van Bruggen, Penetration and delivery characteristics of repetitive microjet injection into the skin. J. Control Release 234, 98 (2016)

11. S. Kaushik, A.H. Hord, D.D. Denson, D.V. McAllister, S. Smitra, M.G. Allen, M.R. Prausnitz, Lack of pain associated with microfabricated microneedles. Anesth. Analg. 92, 502 (2001)

12. F.P.W. Melchels, M.A.N. Domingos, T.J. Klein, J. Malda, P.J. Bartolo, D.W. Hutmacher, Additive manufacturing of tissues and organs. Prog. Polym. Sci. 37, 1079 (2012)

13. Y. Tagawa, N. Oudalov, A.E. Ghalbzouri, C. Sun, D. Lohse, Needle-free injection into skin and soft matter with highly focused microjets. Lab Chip 13, 1357 (2013)

14. B.H. In'T Veld, L. Overmeyer, M. Schmidt, K. Wegener, A. Malshe, P. Bartolo, Micro additive manufacturing using ultra short laser pulses. CIRP Ann. 64, 701 (2015)

15. E. Turkoz, A. Perazzo, H. Kim, H.A. Stone, C.B. Arnold, Impulsively induced jets from viscoelastic films for high-resolution printing. Phys. Rev. Lett. 120, 074501 (2018) 
16. B. Guillotin, A. Souquet, S. Catros, M. Duocastella, B. Pippenger, S. Bellance, R. Bareille, M. Rémy, L. Bordenave, J. Amédée, F. Guillemot, Laser assisted bioprinting of engineered tissue with high cell density and microscale organization. Biomaterials 31, 7250 (2010)

17. R. Xiong, Z. Zhang, W. Chai, Y. Huang, D.B. Chrisey, Freeform drop-on-demand laser printing of 3D alginate and cellular constructs. Biofabrication 7, 045011 (2015)

18. M. Duocastella, J.M. Fernández-Pradas, J.L. Morenza, P. Serra, Time-resolved imaging of the laser forward transfer of liquids. J. Appl. Phys. 106, 084907 (2009)

19. C. Boutopoulos, I. Kalpyris, E. Serpetzoglou, I. Zergioti, Laserinduced forward transfer of silver nanoparticle ink: time-resolved imaging of the jetting dynamics and correlation with the printing quality. Microfluid Nanofluid 16, 493 (2013)

20. P. Serra, M. Duocastella, J.M. Fernández-Pradas, J.L. Morenza, Liquids microprinting through laser-induced forward transfer. Appl. Surf. Sci. 255, 5342 (2009)

21. C.B. Arnold, P. Serra, A. Piqué, Laser direct-write techniques for printing of complex materials. MRS Bull. 32, 23 (2007)

22. B. Guillotin, S. Catros, F. Guillemot, Laser Technology in Biomimetics (Springer, Berlin, 2014), pp. 193-209

23. J.A. Barron, D.B. Krizman, B.R. Ringeisen, Laser printing of single cells: statistical analysis, cell viability, and stress. Ann. Biomed. Eng. 33, 121 (2005)

24. R. Devillard, E. Pages, M.M. Correa, V. Keriquel, M. Rémy, J.Ô. Kalisky, M. Ali, B. Guillotin, F. Guillemot, Methods Cell Biol., Elsevier, pp. 159-174 (2014)

25. N.A. Raof, N.R. Schiele, Y. Xie, D.B. Chrisey, D.T. Corr, The maintenance of pluripotency following laser direct-write of mouse embryonic stem cells. Biomaterials 32, 1802 (2011)

26. S. Young, M. Wong, Y. Tabata, A.G. Mikos, J. Control Release (Rice University, Houston, 2005), pp. 256-274

27. V. Menezes, S. Kumar, K. Takayama, Shock wave driven liquid microjets for drug delivery. J. Appl. Phys. 106, 086102 (2009)

28. X.H. Wang, Y.N. Yan, Y.Q. Pan, Z. Xiong, H.X. Liu, B. Cheng, F. Liu, F. Lin, R.D. Wu, R.J. Zhang, Q.P. Lu, Generation of threedimensional hepatocyte/gelatin structures with rapid prototyping system. Tissue Eng. 12, 83 (2006)

29. C.N. Grover, R.E. Cameron, S.M. Best, Investigating the morphological, mechanical and degradation properties of scaffolds comprising collagen, gelatin and elastin for use in soft tissue engineering. J. Mech. Behav. Biomed. Mater. 10, 62 (2012)

30. L. Möller, A. Krause, J. Dahlmann, I. Gruh, A. Kirschning, G. Dräger, Preparation and evaluation of hydrogel-composites from methacrylated hyaluronic acid, alginate, and gelatin for tissue engineering. Int. J. Artif. Organs 34, 93 (2011)

31. T.J. Hinton, Q. Jallerat, R.N. Palchesko, J.H. Park, M.S. Grodzicki, H.J. Shue, M.H. Ramadan, A.R. Hudson, A.W. Feinberg, Three-dimensional printing of complex biological structures by freeform reversible embedding of suspended hydrogels. Sci. Adv. 1, e1500758 (2015)

32. S. Preibisch, S. Saalfeld, P. Tomancak, Globally optimal stitching of tiled 3D microscopic image acquisitions. Bioinformatics 25, 1463 (2009)

33. V. Dinca, A. Patrascioiu, J.M. Fernández-Pradas, J.L. Morenza, P. Serra, Influence of solution properties in the laser forward transfer of liquids. Appl. Surf. Sci. 258, 9379 (2012)

34. P. Delrot, M.A. Modestino, F. Gallaire, D. Psaltis, C. Moser, Inkjet printing of viscous monodisperse microdroplets by laser-induced flow focusing. Phys. Rev. Appl. 6, 024003 (2016)

35. T. Braschler, A. Valero, L. Colella, K. Pataky, J. Brugger, P. Renaud, Link between alginate reaction front propagation and general reaction diffusion theory. Anal. Chem. 83, 2234 (2011)

36. J. Baxter, S. Mitragotri, Jet-induced skin puncture and its impact on needle-free jet injections: experimental studies and a predictive model. J. Control Release 106, 361 (2005)

37. Z. Zhang, R. Xiong, R. Mei, Y. Huang, D.B. Chrisey, Timeresolved imaging study of jetting dynamics during laser printing of viscoelastic alginate solutions. Langmuir 31, 6447 (2015)

38. L. Koch, A. Deiwick, S. Schlie, S. Michael, M. Gruene, V. Coger, D. Zychlinski, A. Schambach, K. Reimers, P.M. Vogt, B. Chichkov, Skin tissue generation by laser cell printing. Biotechnol. Bioeng. 109, 1855 (2012)

39. B. Guillotin, F. Guillemot, Cell patterning technologies for organotypic tissue fabrication. Trends Biotechnol. 29, 183 (2011)

40. S.V. Murphy, A. Atala, 3D bioprinting of tissues and organs. Nat. Biotechnol. 32(8), 773 (2014)

41. F. Guillemot, A. Souquet, S. Catros, B. Guillotin, J. Lopez, M. Faucon, B. Pippenger, R. Bareille, M. Rémy, S. Bellance, P. Chabassier, J.C. Fricain, J. Amédée, High-throughput laser printing of cells and biomaterials for tissue engineering. Acta Biomater. 6, 2494 (2010)

42. B. Hopp, T. Smausz, N. Kresz, N. Barna, Z. Bor, L. Kolozsvari, D.B. Chrisey, A. Szabo, A. Nogradi, Survival and proliferative ability of various living cell types after laser-induced forward transfer. Tissue Eng. 11, 1817 (2005)

43. B.R. Ringeisen, H. Kim, J.A. Barron, D.B. Krizman, D.B. Chrisey, S. Jackman, R. Auyeung, B.J. Spargo, Laser printing of pluripotent embryonal carcinoma cells. Tissue Eng. 10, 483 (2004) 